\title{
Comparison of Extreme Offshore Structural Response from Two Alterna- tive Stretching Techniques
}

\author{
N.I. Mohd Zaki*,a, M.K. Abu Husain ${ }^{\mathrm{a}}$ and G. Najafian ${ }^{\mathrm{b}}$ \\ ${ }^{a}$ UTM Razak School of Engineering and Advanced Technology, Universiti Teknologi Malaysia, Jalan Semarak, 54100 \\ Kuala Lumpur, Malaysia \\ ${ }^{b}$ School of Engineering, University of Liverpool, L69 3GQ, United Kingdom
}

\begin{abstract}
Linear random wave theory (LRWT) has successfully explained most properties of real sea waves with the exception of some nonlinear effects for surface elevation and water particle kinematics. Due to its simplicity, it is frequently used to simulate water particle kinematics at different nodes of an offshore structure from a reference surface elevation record; however, predicted water particle kinematics from LRWT suffer from unrealistically large high-frequency components in the vicinity of mean water level (MWL). To overcome this deficiency, a common industry practice for evaluation of wave kinematics in the free surface zone consists of using linear random wave theory in conjunction with empirical techniques (such as Wheeler and vertical stretching methods) to provide a more realistic representation of near-surface wave kinematics. It is well known that the predicted kinematics from these methods are different; however, no systematic study has been conducted to investigate the effect of this on the magnitude of extreme responses of an offshore structure. In this paper, probability distributions of extreme responses of an offshore structure from Wheeler and vertical stretching methods are compared. It is shown that the difference is significant; consequently, further research is required to determine which method is more reliable.
\end{abstract}

Keywords: Offshore structure, Wheeler stretching, vertical stretching, Morison's equation, extreme response, Monte Carlo time simulation.

\section{INTRODUCTION}

For an offshore structure, wind, wave and gravitational forces are all important sources of loading. The dominant load, however, is normally due to wind-generated random waves. It is therefore of great importance to calculate the wave loads on the structure accurately. Morison's equation [1] is frequently used to calculate wave loads on the cylindrical members of an offshore structure from the waveinduced water particle kinematics. It can therefore be concluded that accurate estimation of wave-induced water particle kinematics is a key step for accurate prediction of wave loads on the structure.

According to linear random wave theory (LRWT), appropriate transfer functions can be used to calculate water particle kinematics at different nodes of a structure from a reference surface elevation record using the very efficient fast Fourier transform technique. LRWT is a generally acceptable method for determining water particle kinematics below mean water level (MWL) as it is found to predict sensible kinematics. However, water particle kinematics at points above MWL (positive node elevation), calculated from LRWT, suffer from unrealistically large high-frequency

*Address correspondence to this author at the UTM Razak School of Engineering and Advanced Technology, Universiti Teknologi Malaysia Kuala Lumpur, Jalan Semarak, 54100 Kuala Lumpur, Malaysia;

Tel: +603 2203 1402; Fax: +603 2180 5380; E-mail: noorirza@ic.utm.my components (refer to Section 6). A number of empirical techniques have been suggested to provide a more realistic representation of near surface wave kinematics. The empirical techniques popular in the offshore industry include Wheeler stretching [2], linear extrapolation, delta stretching [3], and vertical stretching [4]. Couch and Conte [5] offer a review of these techniques. Each of these modified methods is intended to calculate sensible kinematics in the near surface zone; yet, the predicted kinematics are different leading to uncertainty as to which method should be used.

More accurate results can be obtained from the Hybrid Wave Model, which is a second order random wave theory [6]. In one study [7], water particle kinematics near the free surface zone from some laboratory experiments were compared with predictions from different methods. It was concluded that the Hybrid Wave Model was more accurate than either the Wheeler method or the linear extrapolation technique. The results indicated that while the linear extrapolation method overestimated the water particle kinematics, the reverse was true for the Wheeler method. Longridge et al. [8] made similar conclusions from analysis of laboratory data. They also concluded that both the Wheeler and the linear extrapolation methods are sensitive to the cut-off frequency of the surface elevation frequency spectrum. In other words, they lead to exaggerated water particle kinematics for high-frequency wave components. Donelan et al. [9] also concluded from analysis of laboratory data that both direct application of LRWT and the linear extrapolation method 
greatly overestimate water particle velocities in the near surface zone and that they are both sensitive to the choice of cut-off frequency.

Couch and Conte [5] used water particle kinematics from the Hybrid Wave Model together with those from various stretching techniques to compare the predicted response of the Cognac platform with corresponding measured response data. They concluded that the Hybrid Wave Model leads to more accurate response predictions and that the Wheeler method was better than delta and vertical stretching techniques, which overestimate the response particularly at high frequencies. This conclusion is different from other studies, which indicate that the Wheeler method underestimates the water particle kinematics under wave crests. It should, however, be noted that Morison's drag and inertia coefficients used in this study were 0.90 and 2.3 , respectively, which are somewhat high, and that the response evaluation did not account for variation of wave kinematics in the horizontal direction. The effect of wave directionality was not considered, either. It is, therefore, reasonable to conclude that the response has been overestimated due to the foregoing reasons and that this has compensated for the underestimation of water particle kinematics by the Wheeler method.

Although more data is required to make reliable conclusions, it is generally believed that the Wheeler stretching technique underestimates the water particle kinematics under wave crests while other stretching methods tend to overestimate it. While the industry and the research community are aware of these issues, the impact of this on the magnitude of extreme responses has not been investigated. In this paper, Monte Carlo time simulation technique has been used to investigate the effect of using the Wheeler and the vertical stretching methods of simulating water particle kinematics on the probability distribution of extreme responses. It is shown that the extreme responses from the vertical stretching method could be up to $30 \%$ higher than those predicted from the Wheeler stretching method leading to uncertainty as to which method should be used for design. While it is highly desirable to confirm one of these methods as the preferred one, this cannot be done with high confidence based on the existing literature. It can therefore be concluded that further research, including laboratory and field measurements, is required to determine which of the existing methods is more reliable. Alternatively, new more accurate methods may be suggested. For such a method to be used in practical design, it should also be numerically efficient in derivation of water particle kinematics from a reference surface elevation record.

This paper consists of 9 sections. The following section is devoted to a brief description of the test structure and environmental conditions. Morison's equation for evaluation of wave loading on cylindrical members of offshore structures is briefly discussed in Section 3. Then, a general outline of the simulation of a sample response record is presented in Section 4. Section 5 describes the Monte Carlo time simulation procedure for derivation of the probability distribution of extreme responses. This is followed by a brief description of the Wheeler and the vertical stretching methods for simulation of wave-induced water particle kinematics. Detailed comparison of water particle kinematics from the two methods is offered in Section 7. Probability distributions of the extreme responses from the two different methods of simulating water particle kinematics are compared in Section 8, and finally, the conclusions of this study are summarized in Section 9.

\section{TEST STRUCTURE AND ENVIRONMENTAL CONDITIONS}

In order to keep computational cost to a minimum when simulating a large number of sample records, a simple fixed test structure with four vertical legs, where the diameter of each leg is $1.5 \mathrm{~m}$, in a water depth of $110 \mathrm{~m}$ is used in this study. The dimensions of the platform deck are $35 \mathrm{~m} * 38 \mathrm{~m}$. The general outline of the platform is shown in Fig. (1). As observed, the distributed hydrodynamic load on each leg is represented by 30 point loads so that the total number of nodal loads on the four legs is 120 .

The JCP2, JCP5 and JCP8 structures are used to refer to three finite-element models with first mode natural periods of 2.53, 5.21 and 8.12 seconds, respectively. For JCP2, the Young's modulus of elasticity was taken to be that of normal mild steel $\left(206000 \mathrm{MN} / \mathrm{m}^{2}\right)$. On the other hand, the modulus of elasticity for JCP5 and JCP8 were assumed to be 11220 and $3129 \mathrm{MN} / \mathrm{m}^{2}$, respectively. (It should be noted that the foregoing assumptions, though unrealistic, do not have any impact on the conclusions of this study). For a more complete description of the test structures refer to [10].

The foregoing test structures were subjected to unidirectional random seas simulated from Pierson-Moskowitz (P-M) frequency spectra [11]. The waves were assumed to propagate in the global $\mathrm{Y}$ direction (Fig. 1a). In this study, the following definition of the P-M spectrum has been used:

$G_{\eta \eta}(f)=\frac{H_{S}{ }^{2}}{4 \pi T_{z}^{4} f^{5}} \exp \left(-\frac{1}{\pi T_{z}^{4} f^{4}}\right)$

where $f$ is the wave frequency in $\mathrm{Hz}, G_{\eta \eta}$ is the surface elevation frequency spectrum, $H_{s}$ is the significant waveheight in metres and $T_{\mathrm{z}}$ is the mean zero-upcrossing period in seconds.

The surface elevation and water particle kinematics are calculated according to Linear Random Wave Theory (LRWT). All the water particle kinematics have been multiplied by a wave kinematics factor of 0.95 to account for wave directionality in the sea. The mean zero-upcrossing period (in seconds) for each sea state was taken to be $T_{z}=3.55 \sqrt{H_{s}}$ with $H_{s}$ denoting the significant waveheight in metres. Furthermore, each response has been calculated for three different environmental conditions represented by $H_{s}=15 \mathrm{~m}, 10 \mathrm{~m}$, and $5 \mathrm{~m}$, respectively. Surface elevation frequency spectra for $H_{s}=15 \mathrm{~m}, 10 \mathrm{~m}$ and $5 \mathrm{~m}$ are shown in Fig. (2). The following responses were chosen for investigation: base shear and overturning moment.

\section{MORISON LOADING ON CYLINDRICAL MEM- BERS OF OFFSHORE STRUCTURES}

Morison's equation states that the wave-induced horizontal force per unit length on a vertical submerged cylinder (cylinder diameter / wavelength < 1/5) is the sum of a nonlinear drag component and a linear inertial component. This is shown in the following equation:

$F=F_{d}+F_{i}=k_{d} u|u|+k_{i} \dot{u}$ 


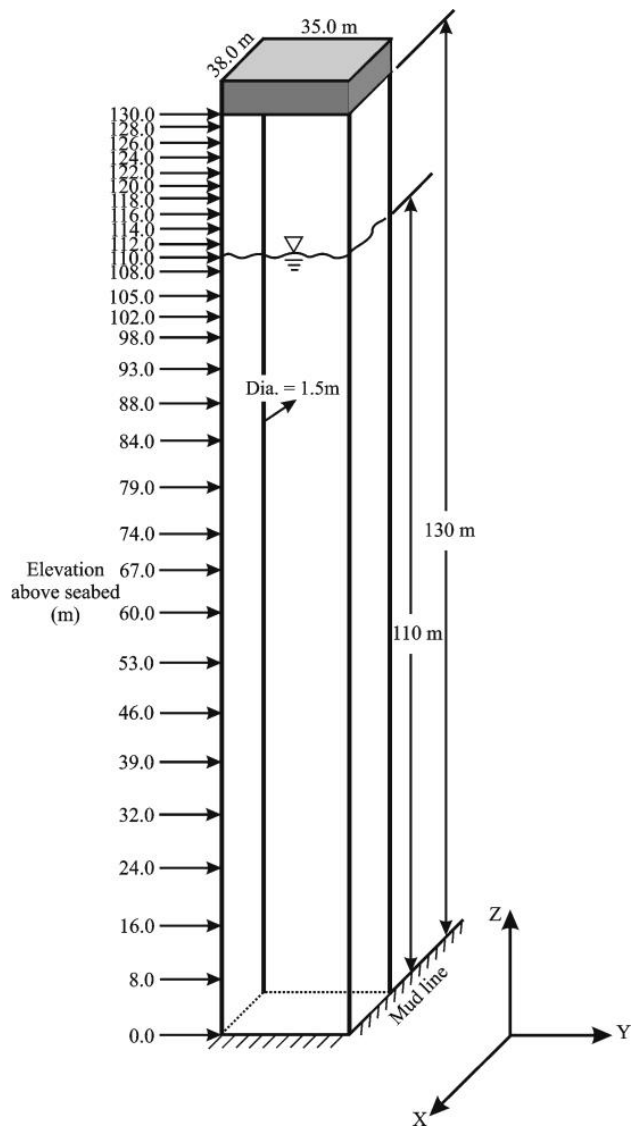

(1a)

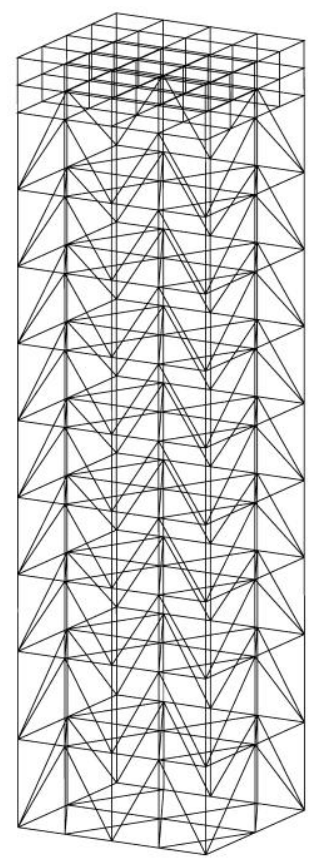

(1b)

Fig. (1). Schematic diagram of the test structures: a) without internal bracings, and b) with internal bracings.

$k_{d}=\frac{1}{2} C_{d} \rho D$

$k_{i}=\frac{1}{4} C_{m} \rho \pi D^{2}(4)$

where $F_{d}$ and $F_{i}$ are the drag and inertial components of fluid loading; $D$ is the cylinder diameter; $\rho$ is the fluid density; $u$ and $\dot{u}$ are the horizontal component of water particle velocity and acceleration at the centre of the cylinder, and $C_{d}$ and $C_{m}$ are empirical drag and inertia coefficients, respectively.

Drag and inertia coefficients are dependent on Reynolds number, Keulegan-Carpenter number and cylinder roughness. In using Morison's equation to calculate the nodal forces, this study assumes that vortex-induced transverse forces have a negligible effect. Further details of the Morison equation can be found in Sarpkaya and Isaacson [12] and Moe [13]. The assumption made in this paper is that Morison's equation with constant $C_{d}$ and $C_{m}$ values can adequately describe the in-line wave forces for a given sea state.

\section{EVALUATION OF QUASI-STATIC AND DYNAMIC RESPONSES THROUGH MODE SUPERPOSITION PROCEDURE}

The steps taken to calculate the dynamic response are as follows:

a. Convert the distributed wave load on each structural element into equivalent point loads at the two ends of the element following the standard procedure in struc-

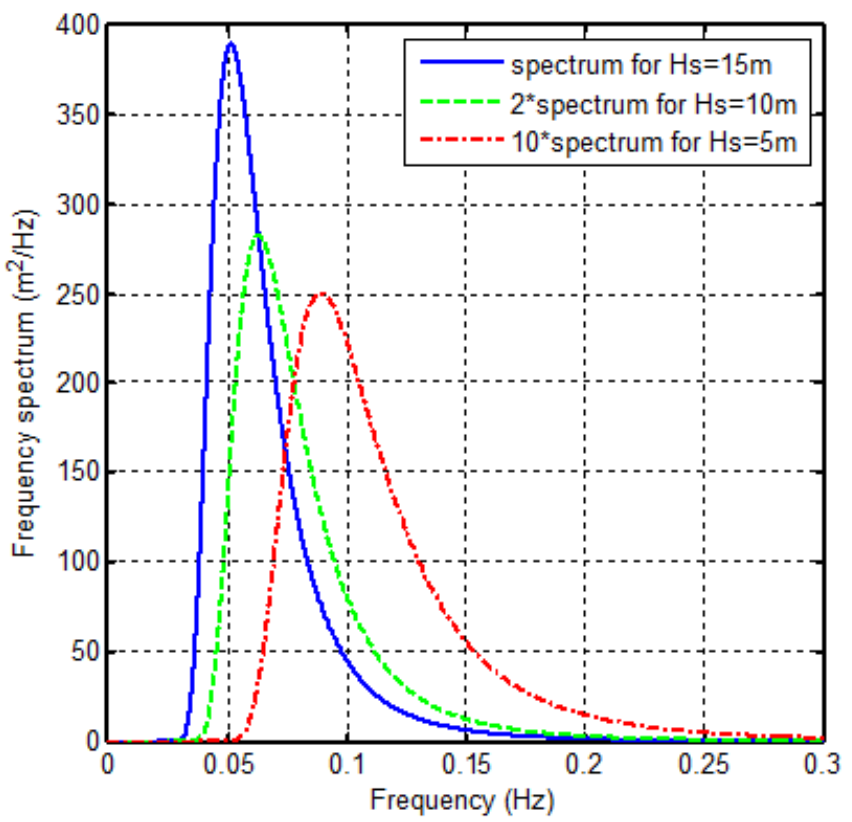

Fig. (2). Water surface elevation frequency spectra for three different sea states. 
tural engineering. (The equivalent point loads are the opposite of reaction forces at the two ends of the element when its two ends are fixed against both displacement and rotation). The equivalent point load at a particular node incorporates the contribution of wave forces on all elements joining each other at the node.

b. Calculate quasi-static response, $\tilde{R}(t)$ as a linear combination of equivalent nodal loads.

$\tilde{R}(t)=\sum_{k=1}^{N} S_{k} p_{k}(t)$

where $S_{k}$ is the flexibility coefficient, $p_{k}(t)$ is the equivalent point load acting on node $k$ incorporating the contribution of wave forces on all the elements joining at the node, and $N$ is the number of nodal forces. The values of $S_{k}$ for a particular response are fixed and are determined from structural analysis.

c. Calculate quasi-static modal amplitudes, $\tilde{Y}_{n}(t)$ as a linear combination of the equivalent nodal loads.

$\tilde{Y}_{n}(t)=\frac{P_{n}(t)}{K_{n}}=\frac{\sum_{k=1}^{N} \phi_{k n} p_{k}(t)}{K_{n}}$

where $P_{n}$ and $K_{n}$ are the $n$th generalised load and generalised stiffness, respectively, and $\phi_{k n}$ is the displacement at node $k$ due to the $n$th mode shape vector. It should be noted that $K_{n}$, is just a number (one-by-one matrix) and that the generalised load, $P_{n}$, is just a linear combination of all the nodal loads.

d. Calculate dynamic modal amplitudes, $Y_{n}(t)$, by applying appropriate frequency response functions (Eq. (7)) to the quasi-static modal amplitudes, $\tilde{Y}_{n}(t)$.

$H_{n}(f)=\frac{1}{1+2 i \varepsilon_{n}\left(\frac{f}{f_{n}}\right)-\left(\frac{f}{f_{n}}\right)^{2}}$

where $f_{n}$ and $\varepsilon_{n}$ are the $n$th mode natural frequency and damping ratio, respectively; $i$ is the imaginary unit and $f$ is the frequency of the excitation $P_{n}(t)$.

e. Compute the difference between the dynamic $(R(t))$ and its corresponding quasi-static response $(\tilde{R}(t))$ from modal analysis.

$[R(t)-\tilde{R}(t)]=\sum_{n=1}^{N M} \delta_{n}\left\{Y_{n}(t)-\tilde{Y}_{n}(t)\right\}$

and

$[\delta]_{1 \times N M}=[\xi]_{1 \times N}[\Phi]_{N x N M}$

where $[\xi]_{1 \times N}$ are the coefficients that relate the response $R(t)$ to nodal displacements, $[\Phi]$ is the mode shape matrix, and $N M$ is the number of modes used in the structural analysis.

f. Calculate the dynamic response using the following equation.

$R(t)=\tilde{R}(t)+[R(t)-\tilde{R}(t)]$

where $\tilde{R}(t)$ is calculated from Eq. (5) and $[R(t)-\tilde{R}(t)]$ is calculated from Eq. (8). Further details of the evaluation of dynamic response of a linear structure through mode superposition procedure can be found in Najafian [10].

\section{DERIVATION OF PROBABILITY DISTRIBUTION OF EXTREME RESPONSES BY THE MONTE CARLO TIME SIMULATION PROCEDURE}

For a short-term distribution (distribution due to a single sea state), use the procedure in Sections 3 and 4 to simulate a response record from a simulated surface elevation record and determine its extreme value. Then repeat the process many times to generate a large sample of extreme responses. Rank all the simulated extreme values from smallest to largest. Then use the following plotting position equation for the Gumbel distribution [14], to estimate the value of the probability distribution for each of the ranked extreme values.

$\operatorname{Prob}\left(r_{\max }<q_{n}\right)=P_{r_{\max }}\left(q_{n}\right) \approx \frac{n-0.44}{N_{s}+0.12}$,

$n=1,2,3, \ldots, N_{s}$

where $r_{\max }$ denotes the response extreme value, $q_{n}$ is the $n$th smallest simulated extreme value, and finally $N_{s}$ is the total number of simulated extreme values.

As an example, the probability distribution of the extreme values of quasi-static base shear of the test structure, plotted to the Gumbel scale, is shown in Fig. (3) for a sea state defined by $H_{s}=15 \mathrm{~m}$ and $T_{z}=13.75 \mathrm{sec}$. The plot is based on 1000 records, each of duration $T=128$ seconds. To demonstrate the important effect of nonlinearities on the probability distribution of extreme responses, the extreme value distribution for an equivalent Gaussian random process (same variance, same $T_{z}$ ) is also shown. It is clear that the Gaussian assumption for response leads to significant underestimation of extreme responses at low probabilities of exceedence; therefore, the Gaussian assumption must be avoided.

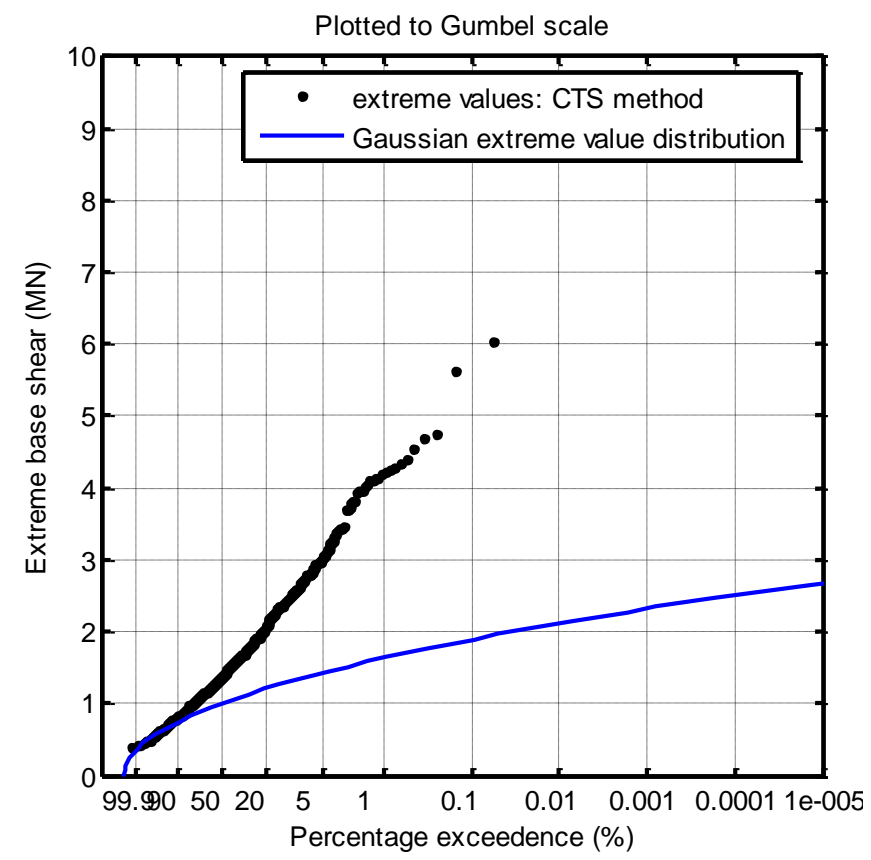

Fig. (3). Probability distribution of extreme values of nonlinear quasi-static base shear. $H_{s}=15 \mathrm{~m}, T_{z}=13.75 \mathrm{sec}, T=128 \mathrm{sec}$, number of response records $=1000$. 
6. VERTICAL AND WHEELER STRETCHING METHODS FOR SIMULATION OF WAVE-INDUCED WATER PARTICLE KINEMATICS

\subsection{Vertical Stretching Method}

According to LRWT, unidirectional seas can be modelled as the sum of a large number of linear progressive wavelets, all travelling in the same direction with random phase angles [15]. The surface elevation at point $x$ and time $t$ can then be expressed as:

$$
\begin{aligned}
& \eta(x, t)=\sum_{i=1}^{N W} \eta_{i}(x, t) \\
& =\sum_{i=1}^{N W} A_{i} \cos \left(2 \pi f_{i} t-k_{i} x-\varphi_{i}\right)
\end{aligned}
$$

where $N W$ is the total number of wavelets used in the simulation, $f_{i}$ are a set of discrete wave frequencies $(\mathrm{Hz})$ and $k_{i}$ are their associated wave numbers. Parameter $\varphi_{i}$ is a random phase angle distributed uniformly in the range $0<\varphi_{i}<2 \pi$, and $A_{i}$ is the amplitude of the $i$ th wave component. The horizontal water particle velocity $(u)$ at a point with elevation $z$ from mean water level (MWL) (assumed to be positive upwards) would then be equal to

$u(x, z, t)=\sum_{i=1}^{N W} u_{i}(x, z, t)$

$u_{i}(x, z, t)=A_{i}\left(2 \pi f_{i}\right) \frac{\cosh \left[k_{i}(d+z)\right]}{\sinh \left(k_{i} d\right)} * \ldots$

$\cos \left(2 \pi f_{i} t-k_{i} x-\varphi_{i}\right)$

where $d$ is the (mean) water depth. For high-frequency components, the wave length would be small and deep water condition would apply; therefore, the above equation can be simplified to

$u_{i}(x, z, t)=A_{i}\left(2 \pi f_{i}\right) e^{k_{i} z} \cos \left(2 \pi f_{i} t-k_{i} x-\varphi_{i}\right)$

The value of $e^{k_{i} z}$ is always smaller than unity for negative $z$ values (points below the MWL); however, it grows very rapidly for high $k$ and $z$ values. This would lead to substantial high-frequency oscillations of water particle kinematics at points above the MWL [16]. As previously mentioned, various techniques have been developed to avoid this problem. The simplest one is the vertical stretching method [4]. In this method, water particle kinematics at points below MWL are calculated from (standard) LRWT, but water particle kinematics above the MWL are taken to be equal to their corresponding values at MWL. In other words, the following relationship is assumed to be valid.

$u(x, z, t)=u(x, 0, t), z>0$

\subsection{Wheeler Stretching Method}

In the Wheeler stretching method [2], the following equation is used to replace the vertical coordinate $z$ with an equivalent node elevation which is always negative. That is,

$z^{\prime}(x, t)=d \frac{d+z}{d+\eta(x, t)}-d$

where $\eta$ is the instantaneous surface elevation at point $x$ and time $t$. It should be clear from the above equation that $z^{\prime}$ changes with time and that it is always negative when the point under consideration is inundated, that is when $\eta>z$. When the surface elevation is below the point, $z^{\prime}$ would be positive, but then, the water particle kinematics must be set equal to zero as the surface elevation is below the point. Therefore, the problem with rapid growth of water particle kinematics for high-frequency wave components would not arise in the case of Wheeler approach. However, since $z^{\prime}$ is a function of time, a transfer function could not be established to convert the surface elevation to water particle kinematics. Therefore, water particle kinematics for each wavelet must be calculated separately, and then, the contributions from all wavelets must be added up to calculate the water particle kinematics due to all wavelets. This is in contrast with the standard LRWT, where a transfer function (and hence the very efficient Fast Fourier Transform technique (FFT)) can be used to calculate water particle kinematics from the reference surface elevation record.

\section{COMPARISON OF WATER PARTICLE KINEMAT- ICS FROM THE WHEELER AND VERTICAL STRETCHING METHODS}

Linear random wave theory was used to simulate a sixhour ( $2^{17}$ data points at $d t=0.1648$ seconds) surface elevation record from a Pierson-Moskowitz surface elevation frequency spectrum with $H_{s}=15 \mathrm{~m}$ and $T_{z}=13.75$ seconds (Fig. 4). Water particle kinematics were then simulated at different node elevations from the two different methods and their values were set equal to zero when the node elevation was above the instantaneous water level. The frequency spectra of water particle kinematics from the two different methods were then compared. The results for water particle velocities and accelerations at a point $3 \mathrm{~m}$ above MWL are shown in Figs. (5 and 6), respectively. As observed in both cases, the frequency spectra from vertical stretching method

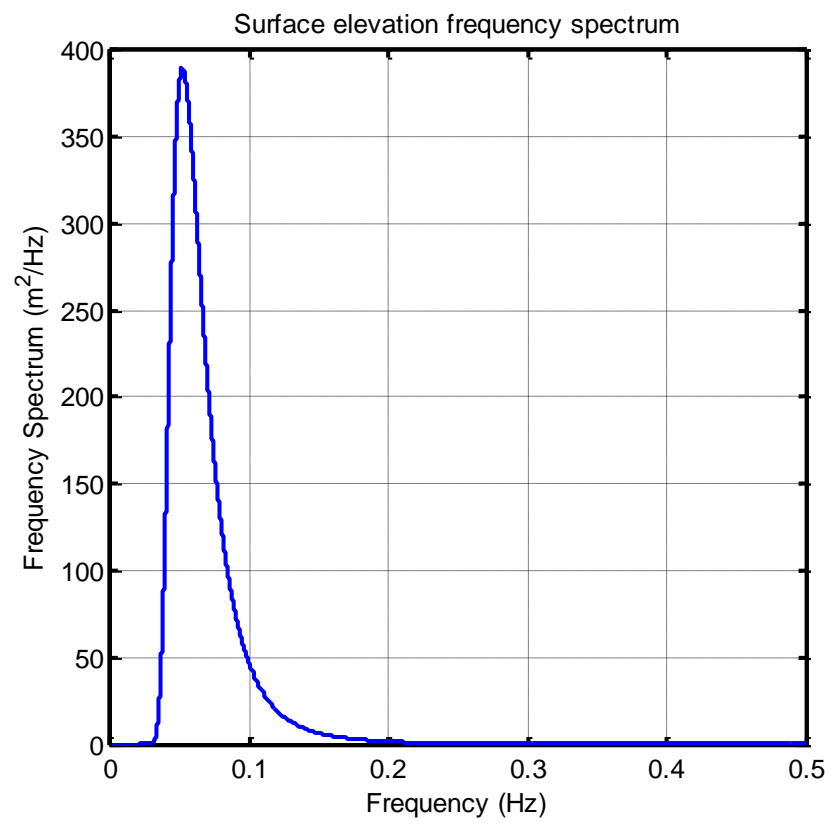

Fig. (4). Pierson-Moskowitz Surface elevation frequency spectrum $\left(H_{s}=15 \mathrm{~m}\right.$ and $\left.T_{z}=13.75 \mathrm{sec}\right)$. 


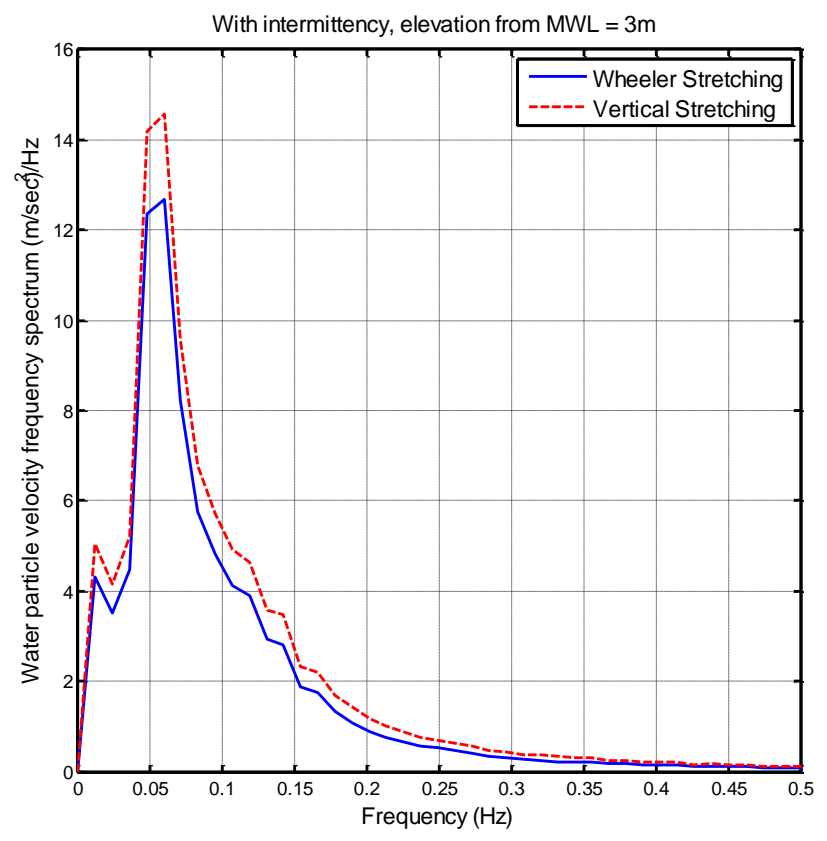

Fig. (5). Frequency spectrum of water particle velocity at a point $3 \mathrm{~m}$ above MWL.

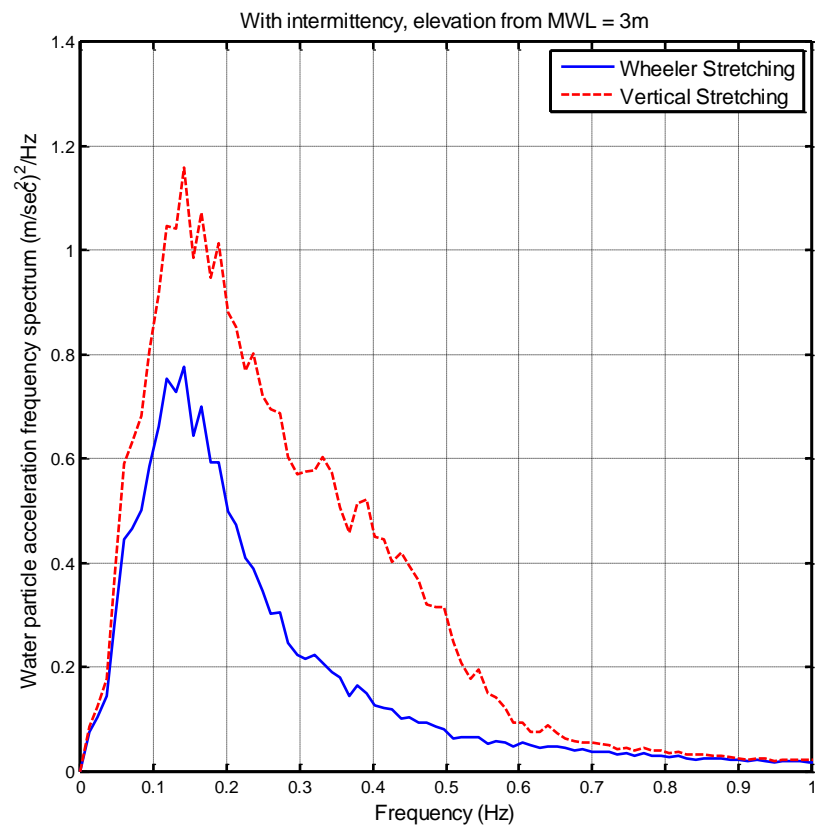

Fig. (6). Frequency spectrum of water particle acceleration at a point $3 \mathrm{~m}$ above MWL.

are higher than those from the Wheeler stretching method in particular for high-frequency components $(f>0.25 \mathrm{~Hz})$ of water particle accelerations.

The largest crest in the simulated surface elevation record was almost $15 \mathrm{~m}$ high (Fig. 7). The corresponding water particle velocities from the two methods at a point $3 \mathrm{~m}$ above mean water level are compared in Fig. (8). As observed, water particle velocities from the Wheeler stretching technique are lower than their corresponding values from the vertical stretching method. Similarly, water particle accelerations from the two methods are compared in Fig. (9). It is clear

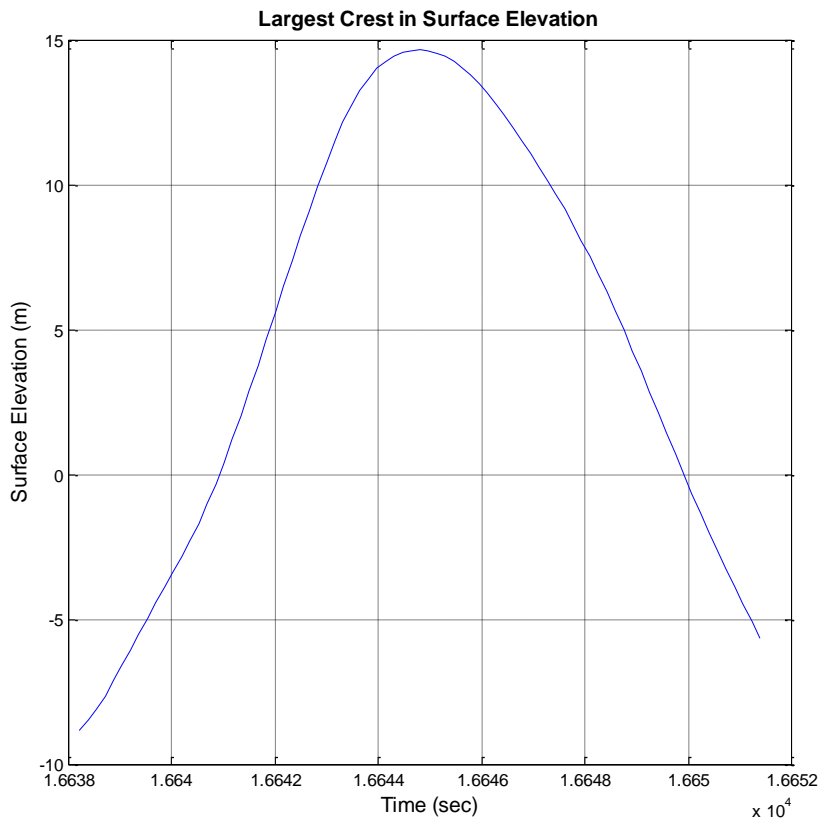

Fig. (7). The largest crest in the simulated surface elevation record.

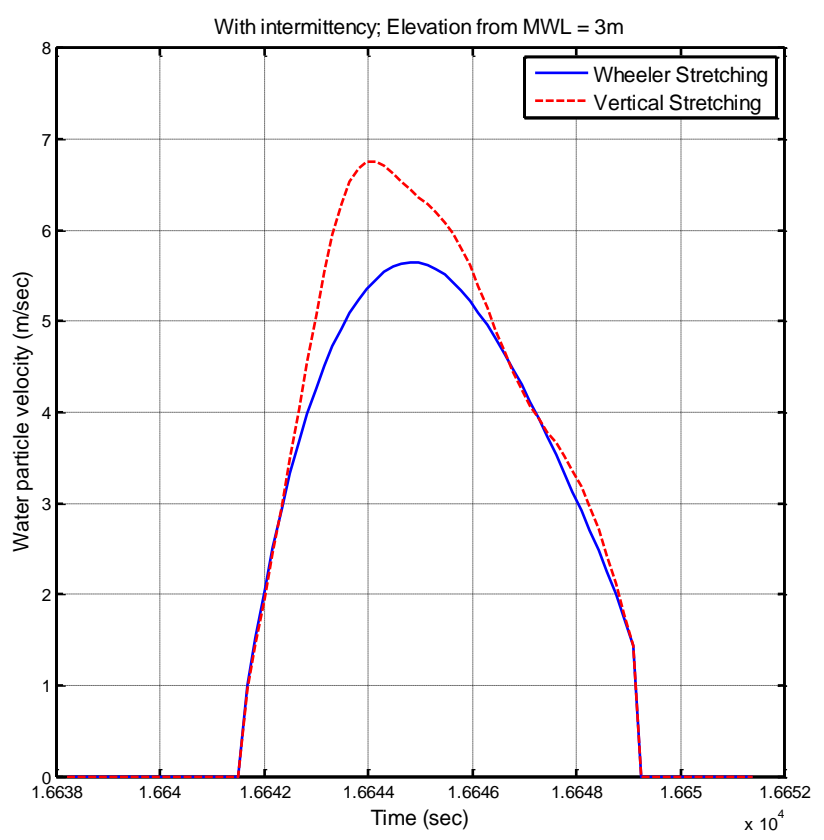

Fig. (8). Water particle velocities from various methods under a very large crest at $3 \mathrm{~m}$ above MWL.

that water particle acceleration from the vertical stretching method is larger and that it has more prominent highfrequency components compared to water particle acceleration from the Wheeler method.

Water particle velocity profiles under the largest crest as a function of node elevation are shown in Fig. (10). It is observed that the velocities from the Wheeler method are systematically smaller than those from the vertical stretching method. In the case of vertical stretching method, the velocity increases rapidly as the MWL is approached from below, but then, it remains constant for all the points above the 


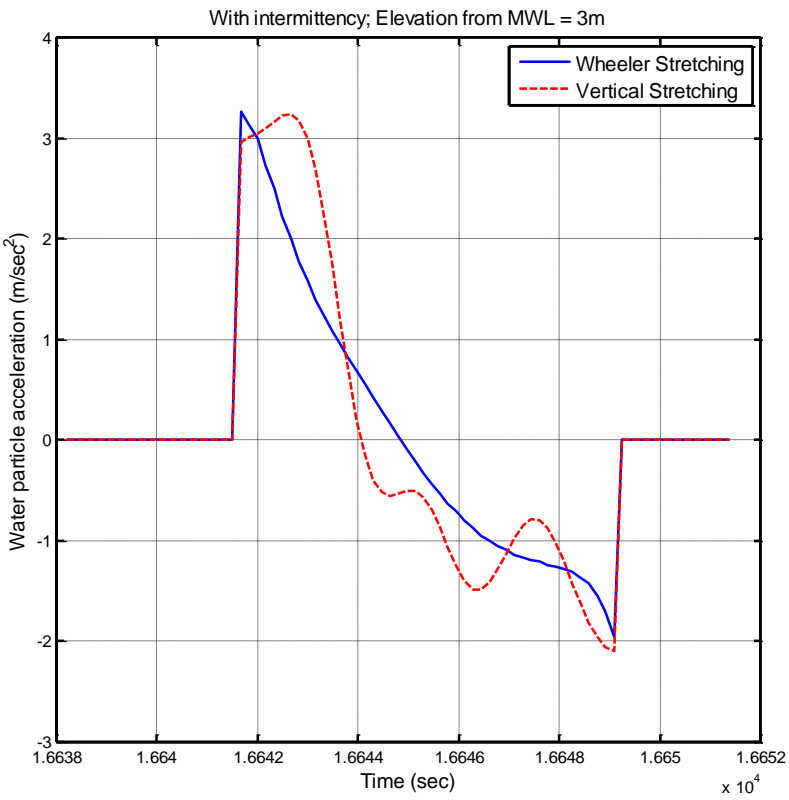

Fig. (9). Water particle accelerations from various methods under a very large crest at $3 \mathrm{~m}$ above MWL.

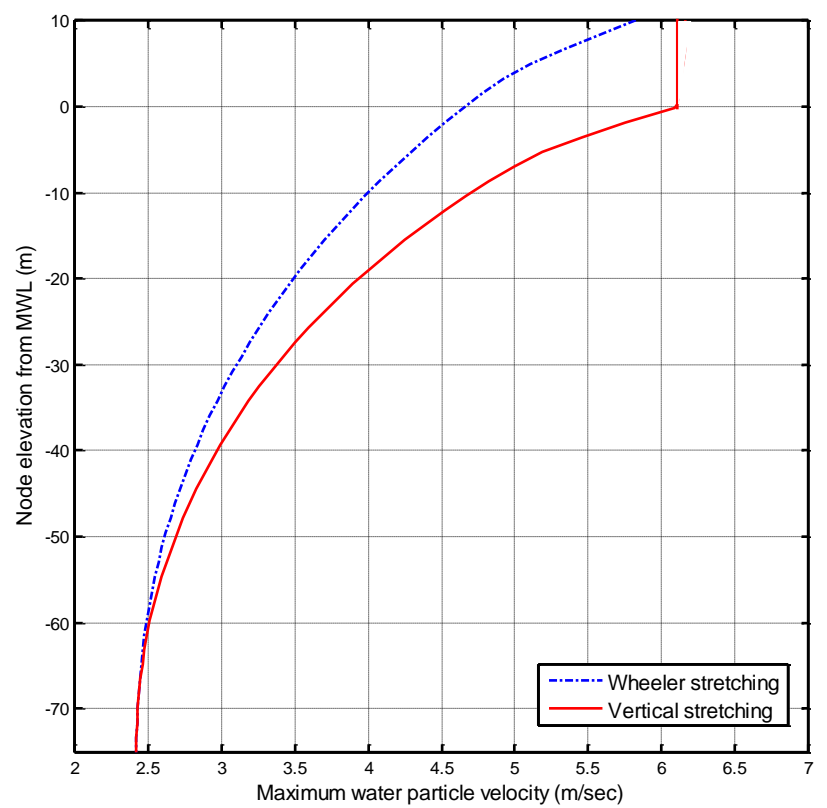

Fig. (10). Comparison of water particle velocity profiles from two different stretching methods.

MWL; in other words, there is an abrupt change in the slope of velocity profile at MWL.

\section{EFFECT OF THE TWO DIFFERENT METHODS OF SIMULATING WATER PARTICLE KINEMATICS ON THE PROBABILITY DISTRIBUTION OF THE EXTREME RESPONSES}

As discussed in Section 2, three different structures and for each structure, two global responses have been investigated. Furthermore, each response has been calculated for three different environmental conditions represented by $H_{s}=$

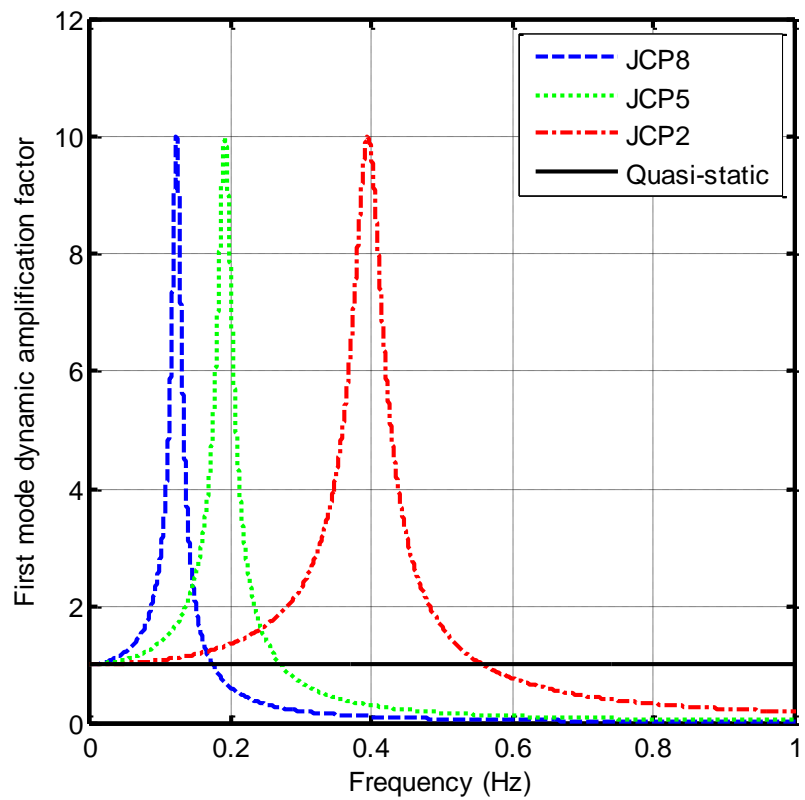

Fig. (11). First mode dynamic amplification factor of the test structures.

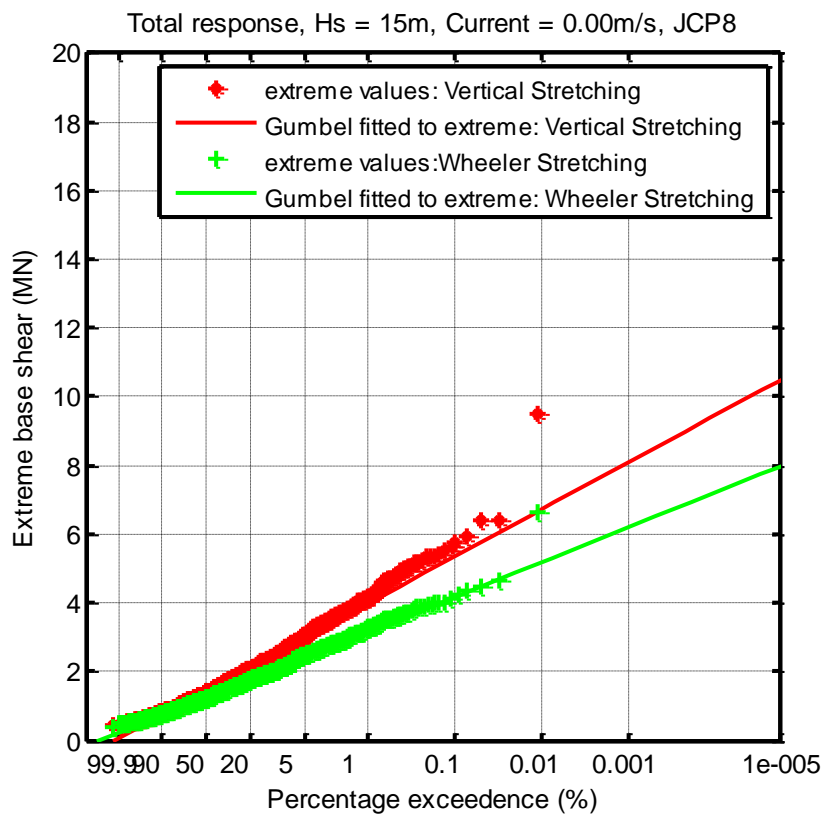

Fig. (12). Comparison of probability distributions of extreme base shears from 2 different methods of simulating water particle kinematics, 1000 sample records, $\mathrm{T}=128 \mathrm{sec}$. JCP8 structure, $H_{s}=$ $15 \mathrm{~m}$, Current $=0.00 \mathrm{~m} / \mathrm{sec}$.

$15 \mathrm{~m}, 10 \mathrm{~m}$, and $5 \mathrm{~m}$, respectively to ensure that the conclusions of this study are valid for a wide range of structures.

The two responses are base shear and overturning moment. The quasi-static response represents the behavior of a very stiff structure for which dynamic effects are negligible. On the other hand, JCP2, JCP5 and JCP8 will represent structures for which dynamic effects are expected to be low, moderate and large, respectively. The first mode dynamic amplification factors for JCP8, JCP5 and JCP2 responses 
together with that for quasi-static responses are shown in Fig. (11).

In this study, the Monte Carlo time simulation technique (Section 5) has been used to derive the probability distributions of the extreme responses from the two different methods of simulating water particle kinematics based on 1000 simulated records, each of $128 \mathrm{sec}$ duration. One thousand simulated extreme values will ensure that the sampling variability is low and would allow any systematic difference between the two distributions to be observed without any ambiguity. Each signal itself is short to reduce the computational effort for the Wheeler method as in this method the very efficient Fast Fourier Transforms cannot be used for evaluation of water particle kinematics from a simulated surface elevation record. It is observed that in all cases (different responses, different $H_{s}$ values and different test structures) the probability distribution of the extreme responses from the vertical stretching method is significantly higher than that from the Wheeler method, leading to uncertainty as to which method should be used for design. As an example, the probability distributions of the extreme base shears from the two methods for $H_{s}=15 \mathrm{~m}$ are compared in Fig. (12). As observed, the difference is not negligible. For example, the extreme base shears at a probability of exceedence of $10^{-7}$ (percentage exceedence of $10^{-5}$ ) from the Wheeler and vertical stretching methods are $7.9 \mathrm{MN}$ and $10.4 \mathrm{MN}$, respectively, indicating that extreme base shear from the vertical stretching method is nearly $32 \%$ higher than that from the Wheeler method. Unfortunately, the existing literature is not sufficient to allow one of these methods to be recommended as the preferred one with a high level of confidence. Further research is, therefore, required to determine which method is more reliable. Alternatively, research may lead to new methods. To be of practical use, a suggested method should be accurate in its predictions, and also efficient in converting a surface elevation record into water particle kinematics.

\section{CONCLUSIONS}

- Linear random wave theory (LRWT) is a generally acceptable method for determining water particle kinematics below mean water level (MWL) as it is found to predict sensible kinematics. However, water particle kinematics at points above MWL (positive node elevation), calculated from LRWT, suffer from unrealistically large high-frequency components. A number of empirical techniques have been suggested to provide a more realistic representation of near surface wave kinematics. The empirical techniques popular in the offshore industry include Wheeler stretching, linear extrapolation, delta stretching, and vertical stretching. Each of these methods is intended to calculate sensible kinematics above the MWL, yet they have been found to differ in their predictions.

- Although it is well known that different methods of simulating water particle kinematics lead to different values of responses, no systematic study has been conducted to investigate the effect of this on the magnitude of the extreme responses.

- The current investigation shows that the probability distributions of extreme responses based on the Wheeler and the vertical stretching methods can be significantly different from each other, leading to uncertainty as to which method should be used in design. Further research is therefore required to resolve this issue.

- Further research may lead to new methods for predicting water particle kinematics in the near surface zone. It would be desirable to compare the results from these methods with high-quality laboratory and field data to observe how well they compare with measured data. Alternatively, they could be compared with water particle kinematics simulated from the more accurate Hybrid Wave Model.

- In this study, the investigation has been carried out based on short simulated records (128 seconds). However, it is commonly assumed that a sea state lasts for a few hours (say 3 hours). This does not cause any problem as the probability distribution of the extreme values during a 3 hour period can be obtained by assuming that the extreme values of successive short segments (128 seconds) are statistically independent from each other.

\section{CONFLICT OF INTEREST}

The author(s) confirm that this article content has no conflicts of interest.

\section{ACKNOWLEDGEMENTS}

This research is financially supported by the Ministry of Higher Education (Malaysia) and Universiti Teknologi Malaysia (UTM) which is gratefully acknowledged.

\section{REFERENCES}

[1] J. R. Morison, M. P. O'Brien, J. W. Johnson, and S. A. Shaaf, "The force exerted by surface waves on piles", AIME Petroleum Transactions, vol. 189, pp. 149-154, 1950.

[2] J. D. Wheeler, "Method for calculating forces produced by irregular waves", Journal of Petroleum Technology, vol. 22, no. 3, pp. 359-367, 1970 .

[3] G. Rodenbusch and G. Z. Forristall, "An empirical model for random directional wave kinematics near the free surface", Proceedings of the 18th Offshore Technology Conference, Houston, Texas, pp. 137-146, 1986.

[4] P. W. Marshall and R. B. Inglis, "Wave kinematics and force coefficients", ASCE Structures Congress, New Orleans, Louisiana, USA, 1986.

[5] A. T. Couch and J. P. Conte, "Field verification of linear and nonlinear hybrid wave models for offshore tower response prediction", Journal of Offshore Mechanical and Arctic Engineering, vol. 119, pp. 158-165, 1997.

[6] J. Zhang, L. Chen, M. Ye, and R. E. Randall, " Hybrid wave model for unidirectional irregular waves, Part I. Theory and numerical scheme", Applied Ocean Research, vol. 18, pp. 77-92, 1996.

[7] C. A. Spell, J. Zhang, and R. E. Randall, "Hybrid wave model for unidirectional irregular waves, Part II. Comparison with laboratory measurements", Applied Ocean Research, vol. 18, pp. 93-110, 1996.

[8] J. K. Longridge, R. E. Randall and J. Zhang, "Comparison of experimental irregular water wave elevation and kinematic data with new hybrid wave model predictions“, Ocean Engineering, vol. 23, pp. 277-307, 1996.

[9] M. A. Donelan, F. Anctil and J. C. Doering, “ A simple method for calculating the velocity field beneath irregular waves", Coastal Engineering, vol. 16, pp. 399-424, 1992.

[10] G. Najafian, "Probability models for offshore structural response due to Morison wave loading, Part I: Drag-only response". Ocean Engineering, vol. 34, no. 17-18, pp. 2277-2288, 2007. 
[11] W. J. Pierson, and L. Moskowitz, “A Proposed Spectral Form for Fully Developed Wind Seas Based on the Similarity Theory of S.A. Kitaigorodskii", Journal of Geophysical Research, vol. 69, pp. 5181-5190, 1964.

[12] T. Sarpkaya, and M. Isaacson, "Mechanics of Wave Forces on Offshore Structures", Van Nostrand Reinhold, New York, 1981.

[13] G. Moe, and R. L. P. Verley, "Hydrodynamic damping of offshore structures in waves and currents", Proceedings of the OTC, pp. 3744, 1980.
[14] G. Lindgren, "Some properties of a normal process near a local maximum", The Annals of Mathematical Statistics, vol. 41, no. 6, pp. $1870-1883,1970$.

[15] L. E. Borgman, "Ocean wave simulation for engineering design", ASCE Journal of Waterways and Harbours Division, 95(WW4), pp. 557-583, 1969.

[16] G. Z. Forristall, "Irregular wave kinematics from a kinematic boundary condition fit (KBCF)", Applied Ocean Research, vol. 7, pp. 202-212, 1985.

(C) Zaki et al.; Licensee Bentham Open.

This is an open access article licensed under the terms of the Creative Commons Attribution Non-Commercial License (http://creativecommons.org/licenses/ by-nc/3.0/) which permits unrestricted, non-commercial use, distribution and reproduction in any medium, provided the work is properly cited. 